\title{
ChemComm
}

\section{Uniaxial negative thermal expansion facilitated by weak host-guest interactions $\dagger$}

Cite this: Chem. Commun., 2014, 50,4238

Received 31st January 2014

Accepted 4th March 2014

DOI: $10.1039 / \mathrm{c} 4 \mathrm{cc} 00849 a$

www.rsc.org/chemcomm

A nitromethane solvate of 18 -crown- 6 was investigated by means of variable-temperature single-crystal $X$-ray diffraction in response to a report of abnormal unit cell contraction. Exceptionally large positive thermal expansion in two axial directions and negative thermal expansion along the third was confirmed. The underlying mechanism relies exclusively on weak electrostatic interactions to yield a linear thermal expansion coefficient of $-129 \times 10^{-6} \mathrm{~K}^{-1}$, the largest negative value yet observed for an organic inclusion compound.

A computational tool developed by van de Streek and Motherwell was used to search the Cambridge Structural Database for previously unidentified polymorphs. Two structures of the nitromethane solvate of 18-crown-6 (18C6N) were flagged (both in the space group $P 2_{1} / n$ with a host-guest ratio of $1: 2$ ). The authors described the pair not as polymorphs but as an example of "extremely anisotropic unit cell contraction". ${ }^{1}$

Unit cell dimensions for $\mathbf{1 8 C 6 N}$ were first reported by McLachlan $^{2}$ and the single-crystal X-ray structure determined by De Boer and co-workers. ${ }^{3}$ The structure was later redetermined at $22{ }^{\circ} \mathrm{C}$ to greater precision by Rogers $e t$ al., who also investigated the crystal structure at $-150{ }^{\circ} \mathrm{C}$ and concluded that at low and high temperature, the crystal has "essentially the same structure except for the obvious exception of weak $\mathrm{CH}$. O bonds". ${ }^{4}$ Indeed, the two structures reported by Rogers were those identified in the van de Streek and Motherwell polymorph search.

For the present study, crystals of $\mathbf{1 8 C 6 N}$ were prepared by dissolving 18-crown-6 in hot nitromethane, followed by filtering and allowing for slow evaporation of the solvent. A crystal was selected while under mother liquor, removed and its surface immediately coated with epoxy to prevent desolvation. The crystal was then glued to the end of a glass fibre and mounted on a goniometer under a liquid nitrogen stream at $90 \mathrm{~K}$. Full intensity

Department of Chemistry and Polymer Science, University of Stellenbosch, Private Bag X1, Matieland, 7602, South Africa. E-mail: ljb@sun.ac.za

$\dagger$ Electronic supplementary information (ESI) available: Synthesis, experimental methods, summaries of crystallographic data, PXRD diffractograms, computational parameters and thermal analysis. CCDC 984223-984232. For ESI and crystallographic data in CIF or other electronic format see DOI: 10.1039/c4cc00849a datasets were collected and the single-crystal X-ray structures were determined at regular temperature intervals in the range 90-273 K; unit cell dimensions were determined to high precision with e.s.d. values in the range $0.0010-0.0023 \AA$ for axis lengths. The $b$ and $c$ unit cell lengths increased steadily and dramatically with temperature while the $a$ axis contracted from 9.31 to $9.12 \AA$ A. No abrupt changes were observed that might indicate a phase transformation (Fig. S1, ESI $\dagger$ ).

Reports of anomalous thermal expansion have become more frequent in recent years but are dominated by inorganic negative linear and volumetric thermal expansion. Typical examples include silicates and zirconium tungstenates that incorporate strong covalent bonds as well as MOF- 5 and related IRMOFs constructed with metal-ligand coordination bonds. ${ }^{5,6}$ In comparison, organic compounds displaying these properties are still relatively rare, though remarkable examples exist. ${ }^{7}$

The degree of linear thermal expansion has been quantified using a coefficient defined as $\alpha_{1}=\left(l_{T}-l_{0}\right) /\left(l_{0}\left(T-T_{0}\right)\right)$ where $l_{T}$ is the axis length at the final temperature $T$ and $l_{0}$ the axis length at the initial temperature $T_{0}$. Similarly, the coefficient of volumetric thermal expansion is $\alpha_{\mathrm{v}}=\left(V_{T}-V_{0}\right) /\left(V_{0}\left(T-T_{0}\right)\right)$ where $V_{T}$ is the unit cell volume at temperature $T$ and $V_{0}$ the volume at $T_{0}$. An important adjustment is needed for crystal systems where the unit cell axes are not mutually orthogonal. ${ }^{6}$ It is necessary to determine an orthogonal set of axes and to calculate coefficients of linear thermal expansion along these principal axes instead of the crystallographic axes (i.e. a strain calculation). The principal axes are labelled $X 1, X 2$ and $X 3$ and are assigned in order of increasing magnitude.

For 18C6N in the 90-273 K temperature range, principal axes and linear expansion coefficients were calculated using the programme $\mathrm{PASCal}^{8}$ The $X 1, X 2$ and $X 3$ axes are approximately along $[-60-1],[-101]$ and $[0-10]$, respectively. Fig. 1 shows how the principal axis lengths change with increasing temperature. The contraction of $X 1$ is overwhelmingly influenced by changes along the crystallographic $a$ axis and, similarly, the expansion of $X 2$ is dominated by changes in the $c$ direction. Fig. S2 (ESI $\dagger$ ) illustrates the orientation of $X 1$ and $X 2$ with respect to the crystallographic 


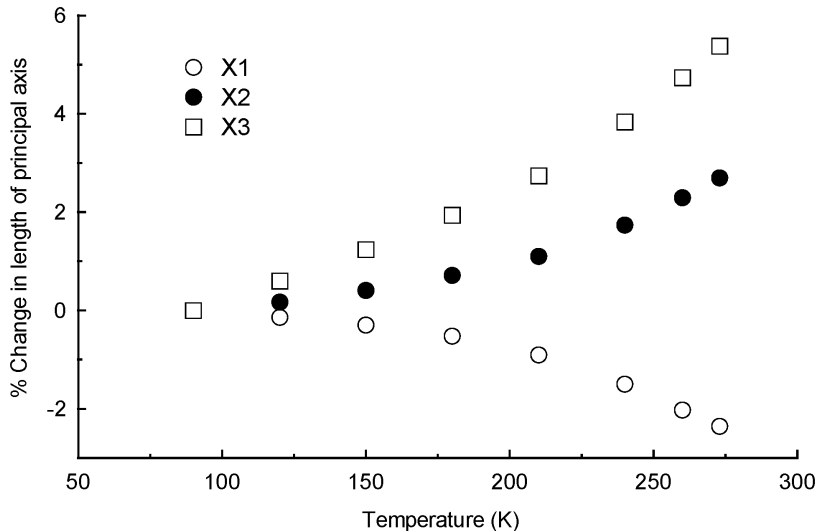

Fig. 1 Percentage changes in principal axis lengths as a function of temperature.

unit cell. The coefficients of linear thermal expansion are $-129(15), 144(14)$ and $282(16) \times 10^{-6} \mathrm{~K}^{-1}$ for $X 1, X 2$ and $X 3$, respectively. The coefficient of volumetric thermal expansion is $311(17) \times 10^{-6} \mathrm{~K}^{-1}$. The negative component of thermal expansion is at least four times greater than that reported recently for another multi-component organic crystal (a complex containing 1,3,5-benzenetricarboxylic acid, 2,6-dimethylaniline and water) and calculated over a similar temperature range $(120-300 \mathrm{~K}){ }^{9}$

A temperature-resolved powder X-ray diffraction experiment (Fig. S3, ESI $\dagger$ ) was carried out by cooling a sample of 18C6N from $293 \mathrm{~K}$ to $123 \mathrm{~K}$ and then heating to $273 \mathrm{~K}$ while collecting data at $30 \mathrm{~K}$ intervals. The results show a gradual, smooth transition between the high and low temperature PXRD patterns as is typical of thermal expansion phenomena that do not involve phase transitions. The absence of $\mathbf{1 8 C 6 N}$ phase transformations was confirmed by a DSC experiment (Fig. S4, ESI $\dagger$ ). Only minor thermal events were identified where residual nitromethane freezes and melts.

Two analogues of $\mathbf{1 8 C 6 N}$ were examined for similar behaviour given that they crystallise with similar structures. The acetonitrile solvate of 18-crown- 6 had already been reported with structures determined at $123 \mathrm{~K}$ and room temperature; the room temperature structure crystallises in the space group $P 2_{1} / n$ (i.e. as does 18C6N) but the low temperature structure is triclinic, suggesting that a temperature-induced phase transformation may occur. ${ }^{10}$ We prepared the iodomethane solvate for the first time and structural data in the temperature range 90-273 $\mathrm{K}$ indicate different degrees of positive thermal expansion along all axes, with the largest coefficient of linear thermal expansion being $129(5) \times 10^{-6} \mathrm{~K}^{-1}$ for $X 3$. However, neither analogue appears to emulate the large negative linear thermal expansion observed over a broad temperature range for $\mathbf{1 8 C 6 N}$. It is therefore clear that the thermal expansion properties of this organic inclusion compound system may be dramatically influenced by changing the guest even if the overall packing arrangement is preserved. Indeed, the expansion along one axis switches from negative to positive by replacing nitromethane with iodomethane. This phenomenon has been alluded to but not previously observed. ${ }^{11}$

Several mechanisms resulting in negative thermal expansion have been described. Arguably the most important of these have involved bridging atoms and rigid unit vibrational modes; magnetostriction; and electronic effects. ${ }^{6,12}$ Das et al. reported extremely large positive and negative thermal expansion for a dumbbell-shaped organic molecule. The mechanism was shown to depend on a helical pattern of strong $\mathrm{O}-\mathrm{H} \cdots \mathrm{O}$ hydrogen bonds. ${ }^{13}$ The 1,3,5-benzenetricarboxylic acid-based compound mentioned earlier is a salt hydrate with an extensive network of ionic and hydrogen bonds. In contrast, an organic polymorph with no ionic interactions or hydrogen bonds was reported recently as undergoing remarkable anisotropic thermal expansion. The mechanism in that case is said to depend on steric effects. ${ }^{14}$ In $18 C 6 N$ there is a similar absence of strong directional intermolecular interactions.

A variety of interactions were investigated computationally for 18C6N (details provided in the ESI $\dagger$ ). The most dominant of these appears to be the host-guest interaction where the guest methyl group is inserted into the crown cavity $\left(-7.16 \mathrm{kcal} \mathrm{mol}^{-1}\right)$ (Fig. S5a, ESI $\dagger$ ). These interactions facilitate the formation of 1:2 host-guest adducts that can be considered as supramolecular building blocks (Fig. S5c, ESI $\dagger$ ). Although their cumulative effect is strongly stabilising, it is mainly weak $\mathrm{C}-\mathrm{H} \cdots \mathrm{O}$ hydrogen bonds that contribute to this interaction. Generally, $\mathrm{C}-\mathrm{H} \cdots \mathrm{O}$ bonds are in the range 3-4 $\AA$ for the donor-acceptor atom distance and $110^{\circ}$ is accepted as a lower limit for the hydrogen bond angle. ${ }^{15}$ The most linear of the hydrogen bonds in $18 \mathrm{C6N}$ is $\mathrm{C} 7-\mathrm{H} \cdots \mathrm{O} 1$ with an angle of $175.4^{\circ}$ and a C-O distance of 3.305(1) Å while the shortest $\mathrm{C}-\mathrm{O}$ contact is $3.132(1) \AA$ in length with a $\mathrm{C}-\mathrm{H} \cdots \mathrm{O}$ angle of $106^{\circ}$ (close to the accepted lower limit).

The next strongest interaction occurs between two guest molecules (of adjacent hosts) which have their dipoles orientated in opposite directions along the $b$ axis (Fig. S5b, ESI $\dagger$ ). The stabilisation energy of this interaction is $-2.22 \mathrm{kcal} \mathrm{mol}^{-1}$. These interactions link adjacent host-guest adducts together in columns parallel to the $b$ axis. Thus a combination of weak host-guest $\mathrm{C}-\mathrm{H} \cdots \mathrm{O}$ hydrogen bonds and long-range dipoledipole stabilisation promotes the formation of columns that dominate the crystal packing (Fig. 2).

Several features contribute to the complex mechanism of negative linear thermal expansion and it is useful to separate these components by considering molecular movement in each of the crystallographic axial planes. First consider the $a b$ plane shown in Fig. 3 (top). The distance between adjacent guest molecules along the $b$ axis increases as expected with increasing temperature.

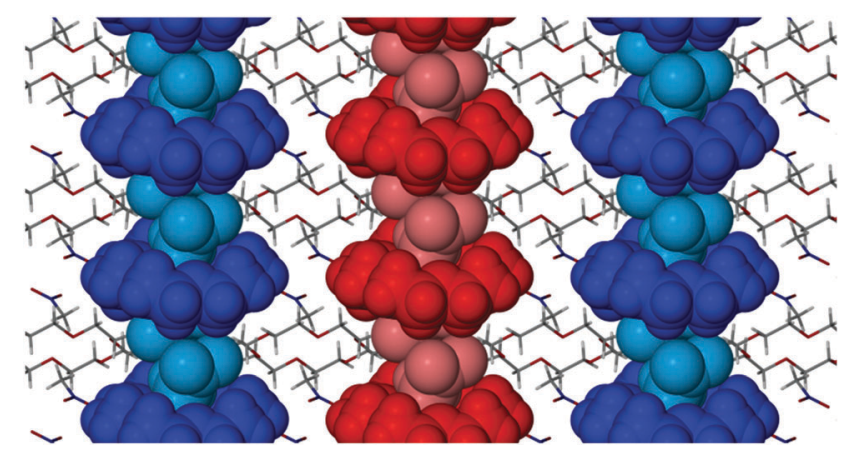

Fig. 2 Packing diagram of $\mathbf{1 8 C 6 N}$ viewed along [100]. Host-guest columns that extend along the crystallographic $b$ axis are shown in blue and red. 

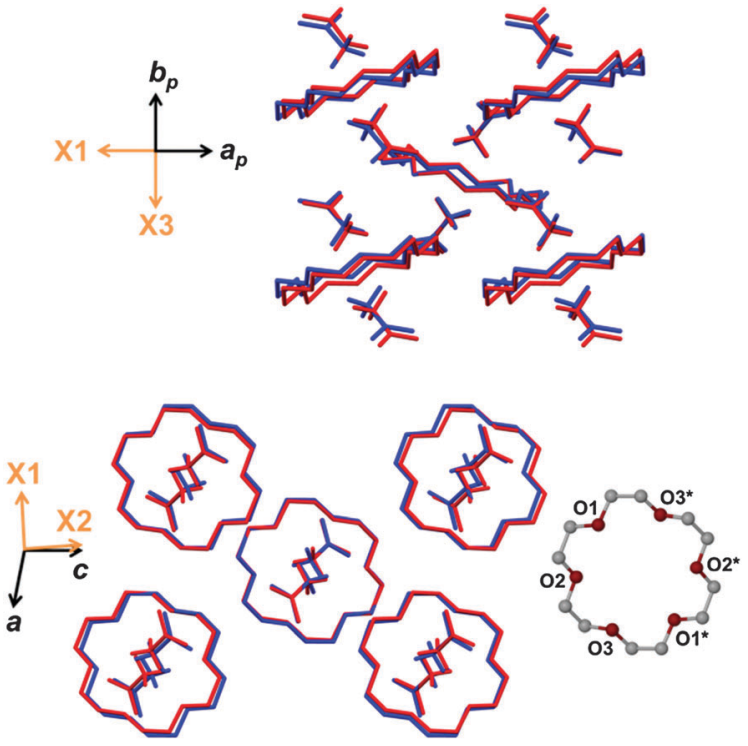

Fig. 3 Projections of the $a b$ (top) and ac (bottom) planes with overlaid structures at $90 \mathrm{~K}$ (blue) and $273 \mathrm{~K}$ (red). Hydrogen atoms have been omitted for clarity.

Similarly the distance between the host and guest centroids increases steadily. In general intermolecular interactions lengthen with increasing temperature because atomic vibrations become larger and because the vibrations are anharmonic. ${ }^{6}$ While the adjacent guests move apart with increasing temperature the host-guest adducts tilt (i.e. the plane of the non-hydrogen atoms of the host crown ether becomes less perpendicular to the $b$ axis). The combined effect of increasing intermolecular distances and tilting of the host-guest adducts accounts for the large expansion of $X 3$.

In the $a c$ plane (Fig. 3, bottom) conformational adjustment of the crown ether molecule is concomitant with the host-guest columns shifting apart along [001] and closer together along [100]. The molecular electrostatic potential maps in Fig. 4 shed light on why the three unique pairs of adjacent columns shift as they do relative to one another. The three maps provide an indication of the relative strength of electrostatic attraction between the columns of each pair. The map of B onto C shows the greatest negative electrostatic potential and the only region of high positive potential, indicating that the $\mathrm{B}-\mathrm{C}$ interaction is
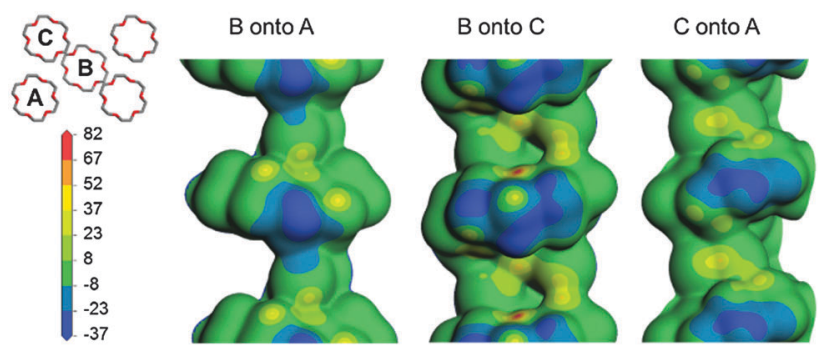

Fig. 4 The molecular electrostatic potential of each column is mapped onto the electron density contour of an adjacent column. The scale bar values are given in $\mathrm{kcal} \mathrm{mol}^{-1}$. the strongest of the three. Since the B-A and C-A interactions are weaker they are more easily overcome when the crystal is heated. While the $\mathrm{B}-\mathrm{C}$ spacing is preserved, the $\mathrm{B}-\mathrm{A}$ and $\mathrm{C}-\mathrm{A}$ distances shrink in response to an increase in temperature and this manifests as a contraction of $X 1$.

Across the centre of the crown ether molecule the $\mathrm{O} 1-\mathrm{O} 1^{*}$ and O3-O3* (* denotes a relationship about a site of -1 symmetry) distances shrink by $0.038 \AA$ and $0.060 \AA$, respectively. These contractions are consistent with the contraction of $X 1$. The O2-O2* distance increases by $0.093 \AA$ and contributes to the expansion of $X 2$. Interestingly, two $\mathrm{C}-\mathrm{H} \cdots \mathrm{O}$ contact distances become shorter as the temperature increases. One of these is the convincing hydrogen bond $\mathrm{C} 7-\mathrm{H} \cdots \mathrm{O} 1$ with a high degree of linearity that is mostly preserved with increasing temperature.

In the $b c$ plane it is clear that the guest methyl rotates as the temperature changes (Fig. S5, ESI $\dagger$ ). The potential for rotation about the $\mathrm{N} 1-\mathrm{C} 7$ bond is the reason the $\mathrm{C} 7-\mathrm{H} \cdots \mathrm{O} 1$ hydrogen bond remains intact and indeed shortens despite an increase in temperature and changes in the host conformation.

Variable temperature structure determination has confirmed that 18C6N undergoes large anisotropic thermal expansion in the temperature range $90-273 \mathrm{~K}$ without any phase transformation. Weak C-H. . O hydrogen bonds facilitate the formation of 1:2 host-guest adducts and dipole-dipole stabilisation allows these adducts to stack as columns. The mechanism giving rise to the negative component of thermal expansion involves anisotropic shifting of host-guest columns relative to one another with simultaneous adjustments in molecular conformation. Negative uniaxial thermal expansion in the absence of strong directional interactions is extremely rare and the case presented here confirms that weak interactions may give rise to this phenomenon. Furthermore, this study raises the exciting prospect that extreme thermal expansion anisotropy in isoskeletal organic inclusion compounds might be controlled by simple variation of the guest, even to the extent of complete reversal. ${ }^{16}$

We are grateful to the SARChI Programme of the Department of Science and Technology and the National Research Foundation (South Africa) for financial support.

\section{Notes and references}

$1 \mathrm{~J}$. van de Streek and S. Motherwell, Acta Crystallogr., Sect. B: Struct. Sci., 2005, 61, 504.

2 R. D. McLachlan, Spectrochim. Acta, Part A, 1974, 30, 2153.

3 J. de Boer, D. Reinhoudt, S. Harkema, G. J. van Hummel and F. de Jong, J. Am. Chem. Soc., 1982, 104, 4073.

4 (a) R. D. Rogers and L. M. Green, J. Inclusion Phenom., 1986, 4, 77;

(b) R. D. Rogers and P. D. Richards, J. Inclusion Phenom., 1987, 5, 631.

5 T. A. Mary, J. S. O. Evans, T. Vog and A. W. Sleight, Science, 1996, 272, 90.

6 G. D. Barrera, J. A. O. Bruno, T. H. K. Barron and N. L. Allan, J. Phys.: Condens. Matter, 2005, 17, R217.

7 (a) M. Zakrzewski and M. A White, J. Phys. Chem., 1990, 94, 2203; (b) H. Birkedal, D. Schwarzenbach and P. Pattison, Angew. Chem., Int. Ed., 2002, 41, 754; (c) R. H. Jones, K. S. Knight, W. G. Marshall, J. Clews, R. J. Darton, D. Pyatt, S. J. Coles and P. N. Horton, CrystEngComm, 2014, 16, 237.

8 J. Cliffe and A. L. Goodwin, J. Appl. Crystallogr., 2012, 45, 1321.

9 S. Bhattacharya and B. K. Saha, Cryst. Growth Des., 2012, 12, 4716.

10 (a) R. D. Rogers, P. D. Richards and E. J. Voss, J. Inclusion Phenom. Mol. Recognit. Chem., 1988, 6, 65; (b) R. L. Garrell, J. C. Smyth, 
F. R. Fronczek and R. D. Gandour, J. Inclusion Phenom. Mol. Recognit. Chem., 1988, 6, 73.

11 I. E. Collings, M. G. Tucker, D. A. Keen and A. L. Goodwin, CrystEngComm, 2014, DOI: 10.1039/C3CE42165A.

12 W. Zhou, H. Wu, T. Yildirim, J. R. Simpson and A. R. Hight Walker, Phys. Rev. B: Condens. Matter Mater. Phys., 2008, 78, 054114.

13 D. Das, T. Jacobs and L. Barbour, Nat. Mater., 2010, 9, 36.
14 S. Bhattacharya and B. K. Saha, CrystEngComm, 2014, 16, 2340.

15 G. Desiraju, Acc. Chem. Res., 1996, 29, 441.

16 (a) G. O. Lloyd, J. Alen, M. W. Bredenkamp, E. J. C. de Vries, C. Esterhuysen and L. J. Barbour, Angew. Chem., Int. Ed., 2006, 45, 5354; (b) L. J. Barbour, D. Das, T. Jacobs, G. O. Lloyd and V. J. Smith, Supramolecular Chemistry: From Molecules to Nanomaterials, John Wiley \& Sons, 2012. 